\title{
Tolerable Acclimation to the Cross-Coupled Illusion through a 10- day, Incremental, Personalized Protocol
}

\author{
Kathrine N. Bretl ${ }^{1 *}$, Aaron T. McCusker ${ }^{1}$, Sage O. Sherman ${ }^{1}$, Thomas R. Mitchell ${ }^{1}$, Jordan B. Dixon ${ }^{1}$, \\ Torin K. Clark ${ }^{1}$
}

BACKGROUND: Artificial gravity (AG) has potential to provide a comprehensive countermeasure

2 mitigating deleterious effects of microgravity. However, the cross-coupled "Coriolis" illusion has

3 prevented using a more feasible and less costly short-radius centrifuge, as compared to large, slowly

4 spinning systems.

5 OBJECTIVE: We assessed tolerability of a personalized, incremental protocol to acclimate humans to the

6 cross-coupled illusion, enabling faster spin rates.

7 METHODS: Ten subjects were exposed to the illusion by performing roll head tilts while seated upright

8 and spun about an Earth-vertical axis. The spin rate was incremented when head tilts did not subjectively

9 elicit the illusion. Subjects completed one 25-minute session on each of 10 days.

10 RESULTS: The spin rate at which subjects felt no cross-coupled illusion increased in all subjects from an

11 average of 1.8 rotations per minute (RPM) (SD: \pm 0.9$)$ at the beginning of the protocol to 17.7 RPM (SD:

$12 \pm 9.1)$ at the end. For off-axis centrifugation producing $1 \mathrm{G}$ at the rider's feet, this corresponds to a reduction

13 in the required centrifuge diameter from 552.2 to 5.7 meters. Subjects reported no more than slight motion

14 sickness.

15 CONCLUSIONS: Acclimation to the cross-coupled illusion, such as that accomplished here, is critical for

16 feasibility of short-radius centrifugation for AG implementation.

17

18 Keywords: artificial gravity, short-radius centrifuge, physiological countermeasure

${ }^{1}$ University of Colorado at Boulder, 1111 Engineering Dr, Boulder, CO 80309

* Corresponding Author, 1111 Engineering Dr. Room 152, Boulder, CO 80309, (303)492-4015,

(303)492-8883, kathrine.bretl@,colorado.edu 


\section{BACKGROUND:}

Artificial gravity (AG) has been considered as a countermeasure for extended-duration

21 human space exploration (e.g., mission to Mars) for over a century [27]. AG provides the potential

22 for a comprehensive countermeasure, in that it can mitigate deconditioning of several physiological

23 systems concurrently (e.g. bone and muscle loss, cardiovascular deconditioning, etc.), in contrast

24 to existing piecemeal countermeasures. Previous approaches to AG have investigated large,

25 slowly rotating centrifuge systems [26]. These concepts have the benefit that humans on-board

26 will likely not be adversely affected by the slow rotation; however, they are technically complex

27 and costly to launch due to the large mass, often considered infeasible for near-term space

28 exploration [4]. An alternative approach to AG is the utilization of a short-radius centrifuge with

29 a diameter on the order of 4-8 meters rather than $100+$ meters, but this requires that the human be

30 spun faster to achieve the desired loading level. This would likely decrease both the mass and cost

31 of the system, but the fast spin rate introduces challenges of its own. The concerns for the human

32 during short-radius centrifugation include 1) the production of a gravity gradient along the body,

33 2) the Coriolis forces associated with moving (e.g., a limb) linearly in the rotating environment,

34 and 3) the disorientation and motion sickness resulting from the cross-coupled (CC) "Coriolis"

35 illusion. Previous research [5,20] leads us to believe that of these three concerns, the limiting factor

36 for short-radius centrifugation is the effect of the $\mathrm{CC}$ illusion on the rider.

37 Further detail is provided elsewhere [11,28], but in summary: the CC illusion is a tilting or

38 tumbling sensation felt by an individual in a constantly rotating environment when executing a

39 head tilt outside of the plane of rotation [11]. The unusual and unexpected stimulation to the

40 vestibular system is highly disorienting and leads to motion sickness. The CC illusion intensity is

41 dependent on the spin rate of the rotating system, head tilt angular velocity, head tilt direction 
42 relative to the spin axis [24], and the angle of the head tilt [13]. Of greatest operational concern

43 for future short-radius centrifugation is the more provocative side effects associated with the faster

44 spin rates required to produce desired loading levels. Investigations from the 1960's assessing

45 humans in a continuously rotating room (up to 10 rotations per minute (RPM)) for extended

46 periods of time (up to 12 days) suggested that a slow spin rate of only a few RPMs was tolerable

$47[9,10,15]$. Faster spin rates led to disorientation and severe motion sickness when head tilts were

48 performed. These adverse effects of the CC illusion at faster spin rates, coupled with the technical

49 challenges of larger AG systems, motivate the development of a protocol to raise tolerance to the

50 illusion, thus enabling a shorter-radius centrifuge.

51 Several studies have shown a reduced illusory response to the CC illusion through repeated

52 exposure $[2,3,12]$. More than one potential physiological mechanism may be involved:

53 "adaptation" (a central reinterpretation of the sensory cues to be more appropriate for the novel,

54 rotating environment) and/or "habituation" (a reduced sensitivity to unexpected sensory cues

55 produced from the novel, rotating environment), and both terms have been used previously. Our

56 approach and findings do not specifically focus on (or depend upon) one or the other or a

57 combination of mechanisms, so here we refer to reductions in CC illusion responses more 58 generally as "acclimation".

59 Acclimation to the $\mathrm{CC}$ illusion has been demonstrated across various subjective measures

60 (e.g. sense of illusory tilt, CC illusion intensity, and motion sickness reports) [31]. Previous CC

61 illusion acclimation studies typically investigated subjects performing a series of head tilts at a

62 spin rate that remained constant throughout the testing session, with each lasting 30-60 minutes,

63 and were repeated once daily for 2-3 consecutive days [2,3,12,29,31]. Acclimation continued over

64 multiple sessions, but even in the longest published experiment to date (5 days), the acclimation 
65 was not complete (i.e., subjects still experienced some CC illusion at the conclusion of the study)

66 [13]. Further, the constant spin rate was often quite fast (e.g., 23 RPM), which elicited severe

67 motion sickness in many of the subjects and resulted in high subject dropout rates (typically 25-

$6835 \%$, even after screening out subjects most susceptible to motion sickness) $[2,12,31]$.

69 To potentially reduce the severity of motion sickness, an incremental approach was

70 proposed where the spin rate (and thus the intensity of the $\mathrm{CC}$ illusion stimulus) was increased

71 over time [23]. For example, one study exposed subjects to 14 RPM on day 1, 23 RPM on day 2,

72 and 30 RPM on day 3 [7]. This approach tended to reduce the proportion of subject dropouts due

73 to severe motion sickness ( $\sim 16 \%$ dropout proportion), as compared to the high-intensity, constant

74 exposure approach.

75 Building upon the incremental approach, it was found that motion sickness could be almost

76 entirely avoided with the use of a threshold-based, or personalized, incremental acclimation

77 approach. In the personalized protocol, the spin rate was incremented based on each individual

78 subject's reporting of the presence or absence of the $\mathrm{CC}$ illusion [3]. In this study [3], supine

79 subjects spun about their roll axis and performed yaw head tilts. The spin rate began at 3 RPM,

80 and once subjects reported no longer feeling the illusion, the spin rate was incrementally increased

81 by 1.5 RPM over 15 seconds. Head tilts were self-paced, but subjects were asked to pause for at

82 least 10 seconds between head tilts to report any $\mathrm{CC}$ illusion. The study found increases in the CC

83 illusion "threshold" both within each 25-minute session, as well as across two sessions on

84 consecutive days. Further, when the $\mathrm{CC}$ illusion was only presented at or near the subject's

85 threshold using the personalized, incremental protocol, 0 of 10 subjects dropped out due to motion

86 sickness. The authors concluded that this threshold-based, incremental procedure is the "method

87 of choice for benign exposure" to the CC illusion [3]. 
Previous studies have noted "individual differences appeared to be rather large" in CC

89 illusion acclimation [3]. However, to date, studies have only reported means across subjects and

90 not specifically quantified inter-individual differences in CC illusion acclimation. Further,

91 previous studies have typically had subjects report the CC illusion intensity on 0-10 scale in which

9210 corresponds to the intensity of the illusion on the first head tilt $[2,12,13,18]$. This makes it

93 difficult to compare responses across subjects, since the scale is normalized for each subject.

\section{2. OBJECTIVE:}

95 In this study, we address previous limitations by assessing the tolerability and efficacy of 96 a threshold-based, personalized, incremental protocol to acclimate humans to the CC illusion.

\section{3. METHODS:}

We tested the effectiveness of a 10-day, personalized acclimation protocol in which

99 subjects were exposed to CC illusion stimuli near their threshold (i.e., where the illusion was barely

100 felt or not at all), limiting motion sickness and encouraging benign exposure to the CC illusion.

101 Our protocol consisted of 10 sessions over the course of 2 weeks - the longest investigation of its

102 kind - aimed at understanding the upper limits of CC illusion acclimation. All subjects began the

103 protocol at 1 RPM, and the spin rate was incremented only when a subject reported experiencing

104 no CC illusion following a pair of head tilts performed in the rotating environment (details in

105 Section 3.3.1. below). The protocol was approved by the University of Colorado Institutional

106 Review Board, and all subjects signed a written informed consent form.

$107 \quad 3.1$ Subjects

108 A total of $10(5 \mathrm{M} / 5 \mathrm{~F})$ healthy subjects volunteered to participate in the study. Inclusion 109 criteria encompassed subjects aged 18-40 years old with no known vestibular dysfunction. All of 110 our subjects met these criteria with an average age of 21.4 years (range: 18-24). Subjects were not 
111 recruited or excluded based upon susceptibility to motion sickness or previous experience that

112 would alter their vestibular acclimation ability (e.g., extensive time spent on airplanes, boats,

113 previous centrifuge experience, etc.). None of our subjects were pilots. On the Motion Sickness

114 Susceptibility Questionnaire (MSSQ) $[21,22]$ subjects scored between the $10^{\text {th }}$ and $99^{\text {th }}$ percentile,

115 with a mean MSSQ percentile of 47 (SD: + /- 29.3). Subjects were left naïve to the overall purpose

116 of the study, the hypothesis of CC illusion acclimation, and the protocol for altering the spin rate.

1173.2 Equipment

118 All experiments were completed in the Bioastronautics Laboratory at the University of

119 Colorado Boulder, using the custom-built Human Eccentric Rotator Device (HERD). Subjects

120 were seated upright in a converted racing chair and rotated clockwise in yaw about an Earth-

121 vertical axis, aligned with the subject's longitudinal (rostrocaudal) axis (Fig. 1). Experiments were

122 performed in the dark to isolate vestibular stimulation and keep subjects naïve to the incrementing

123 of spin rate. Subjects were secured with a 4-point harness and were monitored using infrared

124 cameras. Wireless two-way verbal communication was provided between the subject and

125 operators. Additionally, subjects were provided two wireless pushbuttons for entering responses.

126 Head tilts were limited by foam blocks: one located vertically on the left side of the subject's head,

127 and the other placed at a $40^{\circ}$ angle from vertical on the right side of the subject's head to help

128 ensure consistent head tilt angles.

1293.3 Procedure

130 The protocol consisted of 10 sessions (one session per day over the course of no more than

13114 consecutive days to accommodate subject schedules) in which subjects were spun for 25-

132 minutes per session. At each RPM, subjects spun continuously for 30 seconds to allow for the

133 endolymph in the semicircular canals to equilibrate and any sensation of rotation to decay. 
134 Subjects were then asked to perform a head tilt $40^{\circ}$ right ear down and leave their head tilted while

135 reporting if they experienced the $\mathrm{CC}$ illusion as a result of the head tilt. After 30 seconds of

136 maintaining the 'head tilt down' position, subjects were instructed to tilt their head back upright

137 and again report the presence or absence of the illusion. The head tilt down and the head tilt back

138 up constituted one "head tilt pair". Each head tilt was performed over approximately 1 second.

139 Subjects practiced making smooth, $\sim 1$ second head tilts prior to the beginning of testing.

140 Approximate head tilt duration was verified by infrared video monitoring and subjects were

141 notified if their tilts were performed too rapidly or slowly.

\section{$142 \quad$ 3.3.1 Incrementation Protocol}

143 Following each head tilt pair, the spin rate was incremented or maintained based on the

144 subject reporting whether or not they felt the CC illusion. Specifically, subjects were prompted

145 with the following: "We ask you to simply report whether or not you felt the illusion directly after

146 every head tilt. Sometimes it may be hard to tell, but if you feel anything outside of tilting your

147 head normally in a stationary environment, verbally report "yes" and press the "yes" button.

148 Otherwise, report "no" and press the "no" button." If a subject reported that he/she did not feel

149 the illusion on both head tilts of the pair, the spin rate was increased by 1 RPM over 20 seconds.

150 This duration was selected to keep the angular acceleration subthreshold and the subjects more

151 naïve to the modulation of spin rate based on non-vestibular sensory information (e.g. onset of 152 centripetal acceleration in the subjects' legs). If the subject felt the CC illusion on either or both 153 head tilts of the pair, the spin rate was maintained.

154 The testing sequence, shown in Fig. 2, was repeated for the duration of the 25-minute 155 session. Upon the conclusion of the session, subjects were brought to a stop over $\sim 60$ seconds, 156 unbuckled, and free to leave. Between sessions, subject activity was uncontrolled and 
157 unmonitored, though subjects were asked to refrain from consuming alcohol or excessive amounts

158 of caffeine within 12 hours before each testing session.

159 3.3.2 Starting Spin Rate Determination

160 On the first test session, subjects were initially spun up to 10 RPM to introduce the 161 sensations of the CC illusion. Ten RPM was selected and confirmed to induce a provocative, supra-

162 threshold CC illusion in all subjects, such that they would understand what sensation(s) to be 163 attentive to throughout the duration of testing. Following the performance of a head tilt pair at 10

164 RPM, subjects were spun down to 1 RPM over 60 seconds to commence the testing protocol.

165 On subsequent sessions, the starting RPM was the fastest spin rate that yielded no 166 perception of the CC illusion at the beginning of the previous day's session. This approach was 167 intended to start each day's session at a spin rate near, but below the subject's threshold. 168 Occasionally, the subject reported "yes" that he/she felt the CC illusion on the first head tilt pair 169 at the session's starting RPM, in which case the subsequent session was started 1 RPM below the 170 previous starting RPM (with a minimum of 1 RPM).

\section{3.3.3 Motion Sickness Monitoring}

172 Every 5 minutes during each session (i.e., at 5, 10, 15, 20, and 25 minutes into the session),

173 subjects were asked to verbally report their current motion sickness level. A common 0-20 scale

174 was used (0 corresponds to no sense of motion sickness; 20 is on the verge of vomiting) [2].

175 Subjects were instructed that a score of at least 1 should be reported if there was any sense of 176 motion sickness, no matter how slight. This self-reporting intensity scale has been used extensively

177 to quantify motion sickness resulting from the CC illusion $[2,3,7,8,13,24,29,31]$ and has been

178 found to highly correlate with more complex scales that require experimenter monitoring of 179 physiological responses (e.g., pallor) that were not feasible in our protocol [12]. Our a priori 
180 criteria for prematurely stopping a session included a subject reporting a motion sickness rating

181 (MSR) of 10 or more (or by subject request). If a score of 10 or more was reached on a second

182 session, no additional sessions were performed with that subject.

183 3.4 Data Analysis

184 3.4.1 Analyzed Variables

185 Two performance metrics were extracted on each day of testing to quantify acclimation 186 a subject's beginning threshold and a subject's ending threshold, which correspond to the fastest 187 spin rate (RPM) at which no illusion was felt (i.e., subject reporting of "no, no" on the head tilt 188 pair) at the beginning and end of each testing session, respectively. These metrics allowed us to 189 conservatively determine each individual's tolerable spin rate, assuming that if no illusion is even 190 noticed, the spin rate is presumably operationally tolerable. We also were able to use subjects' CC 191 illusion thresholds to quantify inter-individual differences in acclimation, a benefit over the use of 192 illusion intensity ratings.

193 If a subject reported that he/she felt the CC illusion on either head tilt of the first head tilt 194 pair of any session, this resulted in an inability to properly identify the subject's beginning 195 threshold for that session (since there was an absence of spin rate at which no illusion was felt to 196 start the session). Using the starting spin rate described in Section 3.3.2, this only happened on 21 197 of 100 sessions ( 8 of which occurred when starting at the minimum of 1 RPM). In these 21 cases, 198 we used a proxy for the subject's beginning threshold as 1 RPM less than the starting spin rate. 199 These occurrences are specifically noted in Fig. 4A with open/unfilled shapes.

200 Finally, we analyzed subjects' subjective MSR reports to evaluate motion sickness.

$201 \quad 3.4 .2$ Statistical Analysis 
We assessed the hypothesis that our personalized, incremental protocol would facilitate 203 acclimation across sessions in two manners: 1) paired t-tests between CC illusion threshold on 204 session 1 vs. 10 and 2) a one-sample t-test on the "slope" of a linear fit of CC illusion threshold 205 across session 1-10. Both tests were performed using beginning and ending thresholds. While the 206 data in the paired t-tests statistically met normality assumptions (Anderson-Darling and Shapiro207 Wilks), with only 10 subjects, we also performed non-parametric Wilcoxon signed-rank tests. 208 These reached the same conclusions, so we only present the outcome of t-tests below. The 209 difference in CC illusion threshold inter-individual variance on session 1 vs. 10 was assessed with 210 a F-test.

211 For within-session acclimation, we performed paired t-tests between beginning and ending 212 CC illusion threshold, on each of the 10 sessions. We present the results with and without a 213 Bonferroni correction to account for the 10 pairwise comparisons.

214 The dynamics of motions sickness (MSR reports) were quantified by fitting a hierarchical 215 regression with subject as the identifier, and session number and report within session as factors. 216 The data were transformed to ensure the residuals were normally distributed.

217 Finally, Spearman rank tests were used to assess potential predictors of both how quickly 218 a subject would acclimate to the CC illusion (if age, gender, etc. correlated to acclimation rate 219 and/or ending thresholds), and if that subject would become motion sick during the study (if MSSQ 220 score correlated to reported MSR).

221 Statistical tests were performed with R/RStudio or Systat. The required level of 222 significance for all tests was set to $\alpha=0.05$.

\section{4. RESULTS:}

224 4.1 CC Illusion Acclimation Observed in All Subjects 
All 10 subjects completed the study and showed evidence of acclimation both within and across sessions, though large inter-individual differences were observed. Fig. 3 illustrates the

227 progression of an example subject both within (panel A) and across sessions (panel B). As the 228 example Session 5 progressed, the subject increased in spin rate from a beginning threshold of 3 229 RPM to an ending threshold of 8 RPM.

230 Ten session sequences such as that in Fig. 3A compose Fig. 3B, which shows this same 231 subject's progression over all sessions. As a primary finding, both the subject's beginning (gray 232 triangles in Fig. 3B) and ending (black asterisks) threshold increased from sessions 1 to 10.

\section{4.1.1. Acclimation Across the 10 Sessions}

234 On average, subjects' beginning thresholds increased from 1.8 RPM (range: 1-3) on 235 Session 1 to 12.6 RPM (range: 2-30) on Session 10 (Fig. 4A). The beginning threshold was 236 significantly higher on session 10 than on session $1\left(\mathrm{t}(9)=3.8\right.$, diff $=10.8 \mathrm{RPM}$, Cohen's $\mathrm{d}_{\mathrm{rm}}=1.6$, $237 \mathrm{p}=0.004)$. All 10 subjects had a higher beginning threshold on session 10 as compared to session 238 1. Similarly, the ending threshold increased from an average of 4.1 RPM (range: 1-8) on Session 2391 to 17.7 RPM (range: $3-30)$ on Day 10 , yielding a statistically significant increase $(\mathrm{t}(9)=5.4$, diff $240=13.6$ RPM, Cohen's $\left.\mathrm{d}_{\mathrm{rm}}=2.0, \mathrm{p}<0.0005\right)$. Once again, all 10 subjects displayed a higher ending 241 threshold on session 10 than session 1.

242 To further characterize acclimation with an acclimation rate, we applied a linear fit to each 243 subject's CC illusion thresholds as a function of session number (\#1-10). In these linear fits 244 (separate fit for each of beginning and ending threshold), the slope is the acclimation rate 245 (RPM/session) and the y-intercept is the expected CC illusion threshold (either beginning or 246 ending) on session \#1 for each subject. Residuals of each subject's linear fit were found to be 247 normally distributed (Anderson-Darling, Shapiro-Wilks tests, $p=0.14-0.93$ ), suggesting that 
248 acclimation across sessions for each subject was consistent with being linear. Values of the slopes

249 obtained from the linear regressions are displayed in the legends of Fig. 4 (m-values), and both the

250 slopes and intercepts are given in Table 1. A one-sample t-test on the individual regression slopes

251 (acclimation rates) found they were significantly greater than zero for the beginning thresholds

$252(\mathrm{t}(9)=4.3$, mean $=1.1 \mathrm{RPM} /$ session, 95\% CI: 0.5-1.6, Cohen's $\mathrm{d}=1.9, \mathrm{p}=0.002)$ and ending

253 thresholds $(\mathrm{t}(9)=5.7$, mean $=1.5 \mathrm{RPM} /$ session, 95\% CI: 0.9-2.2, Cohen's $\mathrm{d}=2.6, \mathrm{p}<0.0005)$.

254 Positive slope values correspond to an increase in CC illusion threshold across sessions.

\section{4.1.2. Inter-Individual Differences in Acclimation}

256 The slopes in Table 1 highlight the substantial inter-individual differences in acclimation

257 rate (beginning threshold coefficient of variation $=0.74$, ending threshold coefficient of variation

$258=0.55$ ). Further, there is substantial variation in the ending thresholds on session 10 (standard

259 deviation of 9.1 RPM, coefficient of variation $=0.51$ ). As a result of the varying rates of increasing

260 threshold, the variance in threshold on session 10 was significantly larger than that on session 1 ,

261 both for beginning threshold $(\mathrm{F}(9)=0.0096,95 \%$ CI: $0.0024-0.0386, \mathrm{p}<0.0005)$ and ending

262 threshold $(\mathrm{F}(9)=0.0686,95 \%$ CI: $0.0170-0.2761, \mathrm{p}<0.0005)$. In an effort to identify potential

263 predictors of acclimation ability and thus explain these large variances, a Spearman rank

264 correlation test between an individual's age and rate of acclimation was performed but found to

265 not be significant ( $\mathrm{p}=0.63$ ). Similarly, a two-sample t-test between males and females also found

266 gender to not be significant $(\mathrm{p}=0.85)$.

\section{4.1.3. Acclimation Within Each Session}

268 To assess within-session acclimation, we performed paired t-tests between beginning and

269 ending thresholds for each of the 10 sessions (differences shown in Fig. 4C). All 10 of these tests

270 were statistically significant ( $<<0.0005$ to $p=0.003$ ) such that ending thresholds were greater than 
271 beginning thresholds for each session. We note that when Bonferroni corrections were applied to

272 account for the 10 pairwise comparisons, all sessions still reached statistical significance $(p<0.005$,

273 calculated by dividing $\alpha=0.05$ by the 10 pairwise comparisons).

274 Further, we found that the within-session difference between beginning and ending

275 threshold increased with session number. To quantify this, we fit a hierarchical regression with

276 subject as the identifier and session number as the independent variable. To ensure normality and

277 homoscedasticity of the residuals, we log-transformed the difference between beginning (or

278 ending) threshold $(\mathrm{Y})$, as the dependent variable $\left(Y^{\prime}=\log _{10}(Y+1)\right.$, where 1 was added to

279 produce a real number when $\mathrm{Y}=0$ ). The slope of the regression was significantly greater than zero

$280 \quad(\mathrm{k}=0.036, \mathrm{CI}: 0.024-0.048, \mathrm{Z}(89)=5.7, \mathrm{p}<0.0005)$. This is consistent with subjects acclimating

281 more within each session as they experienced more sessions. We note the apparent decrease on

282 session 10 (Fig. 4C) was primarily due to two subjects who reported that they did not feel the

283 illusion for essentially all of the final session, such that the beginning and ending thresholds were

284 nearly the same, yielding a difference near zero.

285 4.2 Motion Sickness Ratings (MSRs) Remained Low in All Subjects

286 Across all sessions and subjects, the MSRs reported by each subject were generally very

287 low. On the standardized scale of 0 to 20, subjects reported an average MSR of 1.06 (SD: $+/-1.1$ ).

288 Fig. 5A shows the average of the 5 MSRs within each session for all 10 subjects over all 10

289 sessions. Fig. 5B alternatively shows the maximum MSR in each of 10 sessions for all 10 subjects.

290 None of our subjects ever reported an MSR of 10 or greater, yielding a $0 \%(0 / 10)$ dropout rate.

291 4.2.1. Dynamics of Motion Sickness Ratings (MSRs) Within and Across Sessions

292 We aimed to test whether session number or report number within each session affected 293 the reports of motion sickness. To yield one MSR per session for each subject, we first averaged 
294 the 5 reports made in each session (Fig. 5A). Due to the long right tail of the distribution of MSRs,

295 these were transformed by $Y^{\prime}=\log _{10}(M S R+1)$ for statistical analysis, where the plus one was

296 required when $\mathrm{MSR}=0$. We then fit a hierarchical regression with subject as the identifier, session

297 number as the independent variable, and $\mathrm{Y}^{\prime}$ as the dependent variable. This found session number

298 to not have a significant effect on transformed MSRs ( $\mathrm{p}=0.07)$. The trend towards significance was

299 mostly due to session 1, in which the MSRs tended to be higher than in the subsequent sessions

300 (session 1 mean MSR across subjects was 2.1, sessions 2-10 mean MSRs ranged from 0.74 to 1.2).

301 If session 1 was excluded, the hierarchical regression found session number to more clearly be not

302 significant $(\mathrm{p}=0.68)$. This suggests that after session 1 , motion sickness remained fairly constant.

303 Since there was not a clear effect of session number, we averaged across all 10 sessions to

304 yield an average MSR for each subject for each of the 5 reports within sessions. Again, we log-

305 transformed the MSRs, as above. Fitting a hierarchical regression, now with report number as the

306 independent variable, yielded a significant effect $(\mathrm{k}=0.039, \mathrm{CI}: 0.023-0.055, \mathrm{Z}(39)=4.9$,

$307 \mathrm{p}<0.0005)$. The coefficient is in terms of the transformed MSRs, so it does not have meaningful

308 units. The MSRs on the first report of each session averaged only 0.61 but increased on each

309 subsequent report to scores of $0.78,1.21,1.31$, and 1.41 . While motion sickness remained low

310 throughout, it slowly increased during each 25-minute session.

311 4.2.2. Individual Differences in Motion Sickness Ratings (MSRs)

312 We performed Spearman rank correlation tests to evaluate if subjects with a higher MSSQ

313 percentile (i.e., those more susceptible) would have higher MSRs during testing but found no

314 significant correlation between 1) each subject's MSSQ and average MSR across each subject's

31550 reports $(\mathrm{p}=0.55)$, and 2) MSSQ and maximum MSR reported by each subject $(\mathrm{p}=0.94)$. 
Additionally, we evaluated whether a subject's ability to acclimate was correlated with

317 his/her MSRs (i.e., did those who acclimated quickly tend to experience higher or lower motion

318 sickness?). Spearman rank correlation tests were performed, and no significant correlation was

319 found between 1) ending threshold on session 10 and average MSR ( $p=0.36)$; 2$)$ ending threshold

320 acclimation rate and average $\operatorname{MSR}(\mathrm{p}=0.88) ; 3)$ ending threshold on session 10 and maximum

321 MSR ( $p=0.81)$; or 4) ending threshold acclimation rate and maximum MSR ( $p=0.96)$.

322 4.3 Additional Findings Regarding Head Tilt Direction

323 While not the focus of our study, similar to previous investigations [2,19], we found a

324 systematic effect of the direction of head tilt eliciting stronger CC illusions. Specifically, when our

325 subjects reported feeling the CC illusion on only one of the two head tilts in a pair, they tended to

326 report feeling it on the head tilt back to upright. Table 2 shows the number and proportion (in

327 parentheses) of head tilt pairs yielding each possible reporting outcome. Of the pairs in which the

328 illusion was felt on only one of the two head tilts (last two columns), the relative proportions are

329 shown in brackets. Eight of 10 subjects reported feeling the illusion significantly more on the head

330 tilt back to upright as compared to the head tilt down $(*, p<0.05), 1$ subject (subject 7 ) felt the

331 illusion on the head tilt down more frequently than the head tilt up $(\uparrow, \mathrm{p}<0.05)$, and 1 subject did

332 not have a directional asymmetry (subject 4).

\section{5. DISCUSSION:}

334 We found that all ten subjects acclimated as a result of the testing protocol. Both beginning

335 and ending thresholds increased for all subjects between the first testing session and the tenth.

336 This is evidence that subjects are capable of acclimating to the $\mathrm{CC}$ illusion within a session, and

337 that this acclimation carries over from one day to the next. Notably, by the tenth session, seven of

338 our ten subjects reached a spin rate of at least 15 RPM in which they did not experience any CC 
illusion. This corresponds to the spin rate required to obtain $1 \mathrm{G}$ loading at the outer edge of an 8-

340 meter diameter centrifuge. This is a dramatic improvement from the beginning threshold on

341 session 1, averaging 1.8 RPM, which would require a $552.2 \mathrm{~m}$ centrifuge to create the same loading

342 level.

\section{5.1. Comparison to Previous Studies}

344 We aimed to identify subjects' CC illusion "threshold". This metric is different from 345 previous approaches in which participants subjectively reported illusion "intensity", magnitude of 346 illusory tilt angle, or measurement of resulting reflexive eye movements (e.g., time constant of 347 decay of slow phase vestibular ocular reflex (VOR)) [29,31], which have a few limitations. First, 348 the relationship between reported illusion intensity and tolerability is unclear and likely depends

349 upon the individual, their motivation, the task, and the duration (and frequency) which must be 350 tolerated, among other factors. Additionally, while an objective measure, erroneous eye 351 movements from the VOR are likely not the limiting factor for tolerability. We suggest that the 352 forced-choice task ("yes, I felt the CC illusion" vs. "no I did not") is an easier psychophysical task 353 than magnitude estimation on a scale anchored to a sensation experienced potentially days prior 354 (0-10 "intensity" scale). We also suggest that the threshold metric better addresses what is most 355 critical to those designing operational AG centrifuge systems: identification of the fastest tolerable 356 spin rate in physical units of RPM (and thus defining the shortest feasible radius).

357 As hypothesized, our personalized, incremental protocol facilitated benign exposure to the 358 CC illusion. All 10 of our subjects completed the protocol, and none of them reported more than 359 slight motion sickness. The reported motion sickness levels were similar to other personalized, 360 incremental studies [3], and much lower than previous high-intensity exposure investigations $361 \quad[2,12,31]$. Notably, the low motion sickness levels reported here were reached without screening 
362 out subjects highly susceptible to motion sickness, as was done in many previous CC illusion

363 acclimation investigations. Our results did show a statistically significant increase across motion

364 sickness reports within each session; however, we note that even the highest average reports were

365 still quite low (Average $\operatorname{MSR}(\mathrm{t}=25 \mathrm{~min})=1.41 / 20)$. If this trend continued, the sessions would

366 need to be much longer (at least several hours) before subjects reached motion sickness levels of

367 operational concern $(10+/ 20)$.

368 5.2. Inter-Individual Differences Prevalent in Acclimation to the CC Illusion

369 The CC illusion threshold metric allowed us to not only identify acclimation trends within

370 a subject, but also to find and characterize differences between subjects. Previous studies only

371 presented means across subjects [2,3,7,12,29,31], so individual differences in capacity to acclimate

372 were not overtly apparent. In the present study, the session 10 ending threshold ranged from 3

373 RPM to 30 RPM across our 10 subjects. One might suspect the observed differences in acclimation

374 may be due to individual differences in decision boundary of what constitutes "yes" vs. "no" when

375 asked if the illusion was experienced. If this were the case, we would expect it to yield similar

376 inter-individual differences across testing sessions. However, all subjects had beginning thresholds

377 between 1 and 3 RPM on session 1 (range=2 RPM or 3x), while ending thresholds on session 10

378 ranged from 3-30 RPM (range=27 RPM or 10x). This increased range after 10 sessions of exposure

379 suggests that a reasonable portion of the inter-individual variation is due to differences in ability

380 to acclimate rather than differences in reporting decision boundary. However, the factors that

381 cause the individual difference in acclimation ability remain unknown. We note that while no

382 subjects reported vestibular dysfunction, an undiagnosed condition could have contributed to this

383 variability. Other potential explanations of variation include gender or age, but our statistical tests

384 showed no differences between groups in either of these categories. However, our experimental 
385 design was not aimed to predict individual variation (i.e., $5 \mathrm{M} / 5 \mathrm{~F}$ was likely not sufficient to 386 identify a gender-based effect, and our subjects were 18-24 years which would not identify an age 387 effect in older individuals), so we cannot yet rule these effects out. Identification of predictors for 388 rate of acclimation remains an open question, critical to future AG applications. Regardless of the inter-individual differences, each subject appeared to acclimate rather

390 linearly across sessions with no apparent plateau in acclimation reached, as evidenced by the linear 391 regression fits (Table 2). This suggests that continued training sessions could lead to additional 392 acclimation. Future work may investigate whether this linear acclimation trend persists beyond 39310 days, such that all subjects (even "slow" acclimators) could reach an operationally relevant spin 394 rate (e.g., 15 RPM) given a sufficient number of training sessions.

\section{5.3. Implications for Future Configurations}

The configuration used in this investigation included an upright chair spinning about a

397 head-centered, Earth-vertical axis (i.e., yaw rotation). This configuration differs from that expected 398 during centrifugation in a few ways. First, to create centripetal acceleration along the rider's 399 longitudinal axis (aimed at replicating loading here on Earth), the spin axis is typically in roll 400 and/or pitch. Further, our subjects made only roll head tilts in one quadrant (head tilt right ear 401 down and back to upright). However, the three roughly orthogonal semicircular canals transduce 402 angular rotation in any direction. Thus, it is reasonable to expect that acclimation in our 403 configuration is representative of that for rotation in different axes with different head tilts. While 404 representative, if an individual acclimates in one axis (e.g., yaw head tilt), that acclimation does 405 not immediately transfer to another axis (e.g., pitch head tilt) [8]. This suggests that acclimation to 406 a complex, operational centrifuge configuration (e.g., 3D head tilts and a changing orientation 407 relative to the spin axis) may require more generalized and potentially longer training protocols. 
408 The second way our configuration differs is that in centrifugation, the rider's head might be off-

409 axis, producing centripetal acceleration stimulus to the otoliths of the vestibular system, which is

410 negligible for our on-axis configuration. However, we note that any configuration here on Earth

411 has the presence of gravity also stimulating the otoliths, which would not be present during

412 centrifugation either on orbit or in transit. Our ground-based results suggest that substantial

413 acclimation to the $\mathrm{CC}$ illusion is feasible through personalized, incremental training. These

414 approaches will eventually need to be validated in microgravity using a human centrifuge in space.

415 There is some evidence that suggests that the $\mathrm{CC}$ illusion is less provocative in a reduced-

416 gravity environment, both on orbit [17] and during parabolic flight $[6,16]$. Thus, our ground-based

417 results may provide a lower bound for the tolerable spin rate. We note that parabolic flight allows

418 for only $\sim 20$ seconds of microgravity at a time [14], which may be insufficient for all vestibular

419 stimuli to have equilibrated prior to head tilts (e.g., we allow 30 seconds between tilts). Further,

420 parabolic flight does not replicate the neurovestibular adaptations to microgravity that astronauts

421 will undergo during extended orbital spaceflight [30]. While further testing in microgravity is

422 needed, we suggest that if 17 RPM is tolerable on Earth, it is reasonable to expect that at least 17

423 RPM is likely to be tolerable in microgravity.

424 5.4. Mechanisms of Acclimation and Implications

425 Our study did not aim to quantify any altered sensorimotor responses (e.g., perception of 426 head tilt, altered VOR) following each spin session. Nonetheless, no subjects exhibited overt 427 sensorimotor impairment (e.g., poor balance or coordination) and no subjects reported motion 428 sickness or disorientation upon session conclusion. This suggests that the acclimation was 429 "context-specific" [25] to some extent, such that subjects could readily switch back to 430 sensorimotor processing appropriate for the non-rotating environment. This contrasts previous 
431 investigations in the 1960s that did find post-rotation after-effects causing decrements in balance

432 and locomotion as a result of constant rotation for days in a slow rotation room (SRR) $[9,10,15]$.

433 A potential explanation is that dual-adaptation is possible with short-duration, intermittent

434 centrifugation. Operationally, this is relevant for on-orbit or in-transit short-duration

435 centrifugation, as it is critical to maintain appropriate sensorimotor function in the stable spacecraft

436 environment between centrifugation sessions. In spaceflight, centrifugation will also require

437 transitioning to and from microgravity and a gravity-rich environment. Investigating tolerance to

438 this transition occurring regularly will require a human centrifuge on orbit.

439 We note that this acclimation may only be beneficial operationally if it can be retained.

440 Preliminary results suggest that subjects are able to retain of most of their gained acclimation for

441 at least thirty days without CC illusion exposure (i.e., normal activity) [1]. Notably, when we

442 administered a shortened 3-day personalized acclimation protocol following a 30-day break,

443 subjects acclimated at a faster rate during these 3 days than they had during their initial 10-day

444 exposure. This would suggest that even if subjects did not retain all of their gained acclimation,

445 they would be able to regain at a more rapid rate.

446 The personalized, incremental protocol developed in this study is a tolerable approach to

447 expose subjects to the CC illusion without eliciting severe motion sickness, as evident by our $0 \%$

448 dropout rate. Future work may investigate whether the efficacy of acclimation and motion sickness

449 tolerability was the result of incremental increases in spin rate or protocol personalization. The

450 personalization aspect (i.e., each subject's spin rate was incremented based upon his/her responses)

451 may be critical for management of motion sickness, especially in highly susceptible subjects.

452 However, personalized acclimation may not always be feasible in an operational setting (e.g., a 
453 single centrifuge being utilized by multiple astronauts simultaneously). Therefore, next steps

454 should assess the efficacy and tolerability of an incremental but non-personalized approach.

\section{6. CONCLUSIONS:}

456 Acclimation to the $\mathrm{CC}$ illusion is critical to the feasibility of future short-radius centrifuge

457 designs for on-orbit or in-transit AG applications. Though there are several challenges associated

458 with short-radius centrifugation, the limiting factor appears to be acclimation to the CC illusion.

459 We developed a personalized protocol to increase all subjects' tolerability of the CC illusion, even

460 those highly susceptible to motion sickness. Subjects acclimated from an average beginning CC

461 illusion threshold of 1.8 RPM to an average ending threshold of 17.7 RPM after 10 sessions over

4622 weeks. This $17.7 \mathrm{RPM}$ is the spin rate required to create $1 \mathrm{G}$ at the feet for a $5.72 \mathrm{~m}$ diameter 463 centrifuge, a realistic size for the interior of a spacecraft. The linear trend of increasing CC illusion 464 threshold suggests that further acclimation is possible, and that all subjects could potentially reach

465 a desired spin rate with ample exposure. Ultimately, this investigation demonstrates that 466 substantial acclimation to the CC illusion is feasible and tolerable. Enabling high-speed rotation 467 is essential for short-radius centrifugation to create AG, a comprehensive countermeasure for 468 mitigating physiological deconditioning, thus enabling near-future, long-duration space 469 exploration.

\section{ACKNOWLEDGEMENTS}

This work was supported by a NASA Space Technology Research Fellowship.

Preliminary results were presented at the following conferences \& scientific meetings:

Human Research Program Investigators’ Workshop 2017

Aerospace Medical Association Scientific Meeting 2017

Human Research Program Investigators' Workshop 2018 
IEEE Aerospace Conference 2018

Aerospace Medical Association Scientific Meeting 2018

\section{REFERENCES}

470 [1] K.N. Bretl, T.R. Mitchell, S.O. Sherman, A.T. McCusker, J.B. Dixon, T.K. Clark, Retention 471 of Cross-Coupled Illusion Training to Allow for a Shorter Radius Space Centrifuge, in: Big $472 \quad$ Sky, MT, 2018.

473 [2] E.L. Brown, H. Hecht, L.R. Young, Sensorimotor aspects of high-speed artificial gravity: I. 474 Sensory conflict in vestibular adaptation, Journal of Vestibular Research. 12 (2002) 271475282.

476 [3] C.C. Cheung, H. Hecht, T. Jarchow, L.R. Young, Threshold-based vestibular adaptation to 477 cross-coupled canal stimulation, Journal of Vestibular Research. 17 (2007) 171-181.

478 [4] G. Clement, A. Bukley, W. Paloski, Artificial Gravity as a Countermeasure for Mitigating 479 Physiological Deconditioning during Long-Duration Space Missions, Frontiers in Systems $480 \quad$ Neuroscience. 9 (2015).

481 [5] A. Diaz, T. Heldt, L.R. Young, Cardiovascular Responses to Artificial Gravity Combined 482 with Exercise, in: IEEE Aerospace Conference Preedings, Big Sky, MT, 2015.

483 [6] P. DiZio, J. Lackner, J. Evanoff, The influence of gravitoinertial force level on oculomotor 484 and perceptual responses to coriolis, cross-coupling stimulation, Aviation, Space, and $485 \quad$ Environmental Medicine. 58 (1987) 218-223.

486 [7] P. Elias, T. Jarchow, L.R. Young, Incremental adaptation to yaw head turns during 30 RPM 487 centrifugation, Experimental Brain Research. 189 (2008) 269-277. 
[8] I. Garrick-Bethell, T. Jarchow, H. Hecht, L.R. Young, Vestibular adaptation to centrifugation does not transfer across planes of head rotation, Journal of Vestibular Research. 18 (2008) $25-37$.

[9] A. Graybiel, R.S. Kennedy, E.C. Knoblock, F.E. Guedry, W. Mertz, M.E. McLeod, J.K. Colehour, E.F. Miller, A.R. Fregly, Effects of exposure to a rotating environment (10 RPM) on four aviators for a period of twelve days, Aerospace Medicine. (1965) 733-754.

494 [10] F.E. Guedry, R.S. Kennedy, C.S. Harris, A. Graybiel, Human performance during two weeks in a room rotating at three rpm, Aerospace Medicine. (1964) 1071-1082.

[11] F.E. Guedry, E.K. Montague, Quantitative Evaluation of the Vestibular Coriolis Reaction, Aerospace Medicine. 32 (1961) 487-500.

498 [12] H. Hecht, E.L. Brown, L.R. Young, Adapting to artificial gravity (AG) at high rotational speeds, in: Life in Space for Life on Earth, Stockholm, Sweden, 2002.

500 [13] T. Jarchow, L.R. Young, Adaptation to head movements during short radius centrifugation, Acta Astronautica. 61 (2006) 881-888.

502 [14] F. Karmali, M. Shelhamer, The Dynamics of Parabolic Flight: Flight Characteristics and 503 Passenger Percepts, Acta Astronautica. 53 (2008) 594-602.

504 [15] R.S. Kennedy, A. Graybiel, Symptomatology during prolonged exposure in a constantly 505 rotating environment at a velocity of one revolution per minute, Aerospace Medicine. (1962) 817-825.

[16] J. Lackner, A. Graybiel, Influence of gravitoinertial force level on apparent magnitude of coriolis cross-coupled angular accelerations and motion sickness, in: Mechanisms, Prediction, Prevention, and Treatment, Williamsburg, Virginia, 1984. 
[17] J.R. Lackner, A. Graybiel, The Effective Intensity of Coriolis, Cross-Coupling Stimulation is Gravitoinertial Force Dependent: Implications for Space Motion Sickness, Aviation, Space, and Environmental Medicine. 57 (1986) 229-235.

[18] F.W. Mast, N.J. Newby, L.R. Young, Sensorimotor aspects of high-speed artificial gravity: II. The effect of head position on illusory self motion, Journal of Vestibular Research. 12 (2003) 283-289.

[19] J. Mateus, J. Cañizales, A.N. Hearn, L.R. Young, Asymmetry in vestibular responses to cross-coupled stimulus, Experimental Brain Research. 209 (2011) 561-569.

[20] T. Piotrowski, J. Rittweger, J. Zange, A Comparison of Squatting Exercise on a Centrifuge and With Earth Gravity, Frontiers in Physiology. 9 (n.d.).

[21] J.T. Reason, Relations Between Motion Sickness Susceptibility, The Spiral After-Effect and Loudness Estimation, British Journal of Psychology. 59 (1968) 385-393.

[22] J.T. Reason, J.J. Brand, Motion Sickness, Academic Press London, 1975.

[23] J.T. Reason, A. Graybiel, Progressive adaptation to Coriolis accelerations associated with 1-rpm increments in the velocity in the slow-rotation room, Aerospace Medicine. 41 (1970) 73-79.

[24] S.E. Sheehan, L.R. Young, T. Jarchow, The effect of head turn velocity on cross-coupled stimulation during centrifugation, Journal of Vestibular Research. 18 (2008) 1-14.

[25] M. Shelhamer, R. Clendaniel, Context-specific adaptation of saccade gain, Experimental Brain Research. 146 (2002) 441-450.

[26] R.W. Stone, W.M. Piland, W. Letko, Certain Aspects of Onboard Centrifuges and Artificial Gravity, in: Fourth Symposium on the Role of the Vestibular Organs in Space Exploration, Naval Aerospace Medical Institue, Pensacola, Florida, 1968: pp. 331-343. 
533 [27] K.E. Tsiolkovsky, The Exploration of the Universe with Reaction Machines, NASA,

$534 \quad$ Washington, D. C., 1911.

535 [28] G. Vincent, J. Gruber, M. Newman, T.K. Clark, Analysis of Artificial Gravity Paradigms

536 using a Mathematical Model of Spatial Orientation, Acta Astronautica. 152 (2018) 602-

$537 \quad 610$.

538 [29] L.R. Young, H. Hecht, L.E. Lyne, K. Sienko, C.C. Cheung, J. Kavelaars, Artificial gravity:

$539 \quad$ Head movements during short-radius centrifugation, Acta Astronautica. 49 (2001) 215-226.

540 [30] L.R. Young, C.M. Oman, D.G. Watt, K.E. Money, B.K. Lichtenberg, Spatial Orientation in

541 Weightlessness and Readaptation to Earth's Gravity, Science. 225 (1984) 205-208.

542 [31] L.R. Young, K.H. Sienko, L.E. Lyne, H. Hecht, A. Natapoff, Adaptation of the vestibulo-

543 ocular reflex, subjective tilt, and motion sickness to head movements during short-radius

544 centrifugation, Journal of Vestibular Research. 13 (2003) 65-77. 
Table 1 - Slopes and Intercepts of Acclimation Linear Regressions

\begin{tabular}{ccccc}
\hline \multirow{2}{*}{ Subject } & \multicolumn{2}{c}{ Slope of Linear Fit (RPM/session) } & \multicolumn{2}{c}{ Intercept of Linear Fit (RPM) } \\
\cline { 2 - 5 } & Beginning Threshold & Ending Threshold & Beginning Threshold & Ending Threshold \\
\hline $\mathbf{1}$ & 0.33 & 0.44 & 0.93 & 3.44 \\
\hline $\mathbf{2}$ & 1.33 & 2.22 & 2.13 & 5.02 \\
\hline $\mathbf{3}$ & 0.19 & 0.87 & 4.12 & 9.00 \\
\hline $\mathbf{4}$ & 0.84 & 1.42 & 0.64 & 2.29 \\
\hline $\mathbf{5}$ & 0.69 & 1.53 & 0.22 & -0.78 \\
\hline $\mathbf{6}$ & 1.02 & 1.62 & -1.31 & 6.63 \\
\hline $\mathbf{8}$ & 1.49 & 1.70 & 0.62 & 4.42 \\
\hline $\mathbf{9}$ & 2.30 & 2.69 & 0.03 & 9.11 \\
\hline $\mathbf{1 0}$ & 2.27 & 2.71 & -0.02 & 0.78 \\
\hline Average & 0.18 & 0.25 & 1.20 & 4.09 \\
\hline Standard Deviation & 1.06 & 1.54 & 1.89 & 3.41 \\
\hline Coefficient of & 0.78 & 0.85 & 1.58 & 0.83 \\
Variation & 0.74 & 0.55 & & \\
\hline
\end{tabular}


Table 2 - Number and Proportion of Head Tilt Pairs Yielding Each Possible Response Outcome

\begin{tabular}{llllll}
\hline Subject & $\begin{array}{l}\text { Number } \\
\text { of Head } \\
\text { Tilt Pairs }\end{array}$ & $\begin{array}{l}\text { Pairs Reported } \\
\text { "Yes" on Tilt Down, } \\
\text { "Yes" on Tilt Up }\end{array}$ & $\begin{array}{l}\text { Pairs Reported } \\
\text { "No" on Tilt Down, } \\
\text { "No" on Tilt Up }\end{array}$ & $\begin{array}{l}\text { Pairs Reported } \\
\text { "Yes" on Tilt Down, }\end{array}$ & $\begin{array}{l}\text { Pairs Reported } \\
\text { "No" on Tilt Up } \\
\text { on Tilt Down, } \\
\text { "Yes" on Tilt Up }\end{array}$ \\
\hline $\mathbf{1}$ & 196 & $62(31.6 \%)$ & $44(22.4 \%)$ & $7(3.6 \%)[7.8 \%]$ & $83(42.3 \%)[92.2 \%] *$ \\
\hline $\mathbf{2}$ & 184 & $19(10.3 \%)$ & $97(52.7 \%)$ & $16(8.7 \%)[23.5 \%]$ & $52(28.3 \%)[76.5 \%]^{*}$ \\
\hline $\mathbf{3}$ & 196 & $28(14.3 \%)$ & $97(49.5 \%)$ & $10(5.1 \%)[14.1 \%]$ & $61(31.1 \%)[85.9 \%] *$ \\
\hline $\mathbf{4}$ & 199 & $77(38.7 \%)$ & $61(30.7 \%)$ & $38(19.1 \%)[62.3 \%]$ & $23(11.6 \%)[37.7 \%]$ \\
\hline $\mathbf{5}$ & 208 & $36(17.3 \%)$ & $64(30.8 \%)$ & $8(3.8 \%)[7.4 \%]$ & $100(48.1 \%)[92.6 \%]^{*}$ \\
\hline $\mathbf{6}$ & 210 & $83(39.5 \%)$ & $55(26.2 \%)$ & $7(3.3 \%)[9.7 \%]$ & $65(30.9 \%)[90.3 \%]^{*}$ \\
\hline $\mathbf{7}$ & 209 & $38(18.2 \%)$ & $104(49.8 \%)$ & $45(21.5 \%)[67.2 \%] \dagger$ & $22(10.5 \%)[32.8 \%]$ \\
\hline $\mathbf{8}$ & 197 & $43(21.8 \%)$ & $100(50.8 \%)$ & $12(6.1 \%)[22.2 \%]$ & $42(21.3 \%)[77.8 \%]^{*}$ \\
\hline $\mathbf{9}$ & 174 & $14(8.0 \%)$ & $98(56.3 \%)$ & $7(4.0 \%)[11.3 \%]$ & $55(31.6 \%)[88.7 \%]$ \\
\hline $\mathbf{1 0}$ & 221 & $155(70.1 \%)$ & $20(9.0 \%)$ & $10(4.5 \%)[21.7 \%]$ & $36(16.3 \%)[78.3 \%]^{*}$ \\
\hline Average & 199.4 & $(27.0 \%)$ & $(37.8 \%)$ & $(8.0 \%)[24.7 \%]$ & $(27.2 \%)[75.3 \%]$ \\
\hline
\end{tabular}




\section{FIGURE CAPTIONS}

Fig. 1: CU's Human Eccentric Rotator Device (HERD), displaying both head positions between which head tilts were made: ‘head tilt up' (Panel A) and 'head tilt down' (Panel B).

Fig. 2: Flow chart of personalized, incremental testing protocol.

Fig. 3: Example subject staircase. Panel A shows the incremental increases in spin rate according to $\mathrm{CC}$ illusion reported after each head tilt. Spin rate was only increased when the illusion was not felt on both head tilts of one head tilt pair. This data represents subject 4 on his/her fifth testing session. This particular subject started testing at 2 RPM on Session 5, which corresponds to his/her beginning threshold from the previous Session 4. Across the bottom of the graph, the individual subject reports of "yes" he/she did feel the illusion (denoted with a "Y"), or "no" he/she did not feel the illusion (denoted with an "N") can be seen for each individual head tilt down (top row) and head tilt up (bottom row). Vertical dashed lines show when the spin rate was incremented, which only occurred following two "N" reports, first on the head tilt down, then on the head tilt up. Panel B shows the spin rate staircase across all 10 sessions for subject 4, showing progression of beginning (gray triangles and connecting lines) and ending (black asterisks and connecting lines) threshold. Each staircase section corresponds to one session of testing. For reference, the required centrifuge diameter to produce 1 Earth $G$ (at the rider's feet) for each spin rate is shown on the right axis (e.g., 8 RPM requires a 28.0 m diameter centrifuge to produce $1 \mathrm{G}$ ).

Fig. 4: Progression of beginning (panel A, top) and ending (panel B, middle) thresholds for all 10 subjects (different shapes and shades of gray) over 10 sessions of personalized, incremental 
acclimation. Open shapes indicate when the beginning threshold could only be estimated, based on subjects reporting that they felt the CC illusion on either or both head tilts of the first head tilt pair. Solid black lines indicate the sample mean. Linear regression fit slope-values (as RPM increase/session) for each subject are provided in the legend as m-values. The right y-axis shows the centrifuge diameter (in meters) required to produce $1 \mathrm{G}$ at the rider's feet, corresponding to the spin rates on the left. Note that the relationship between spin rate and required diameter is nonlinear. Panel C (bottom) shows the average difference between beginning and ending thresholds (averaged across all subjects) for each session. The shaded area shows the $95 \%$ confidence interval across subjects.

Fig. 5: Motion sickness rating (MSR) scores reported within each session with all 10 subjects displayed (100 total scores). Panel A shows average MSR scores reported in each session, and panel B shows maximum MSRs reported. 
Fig. 1
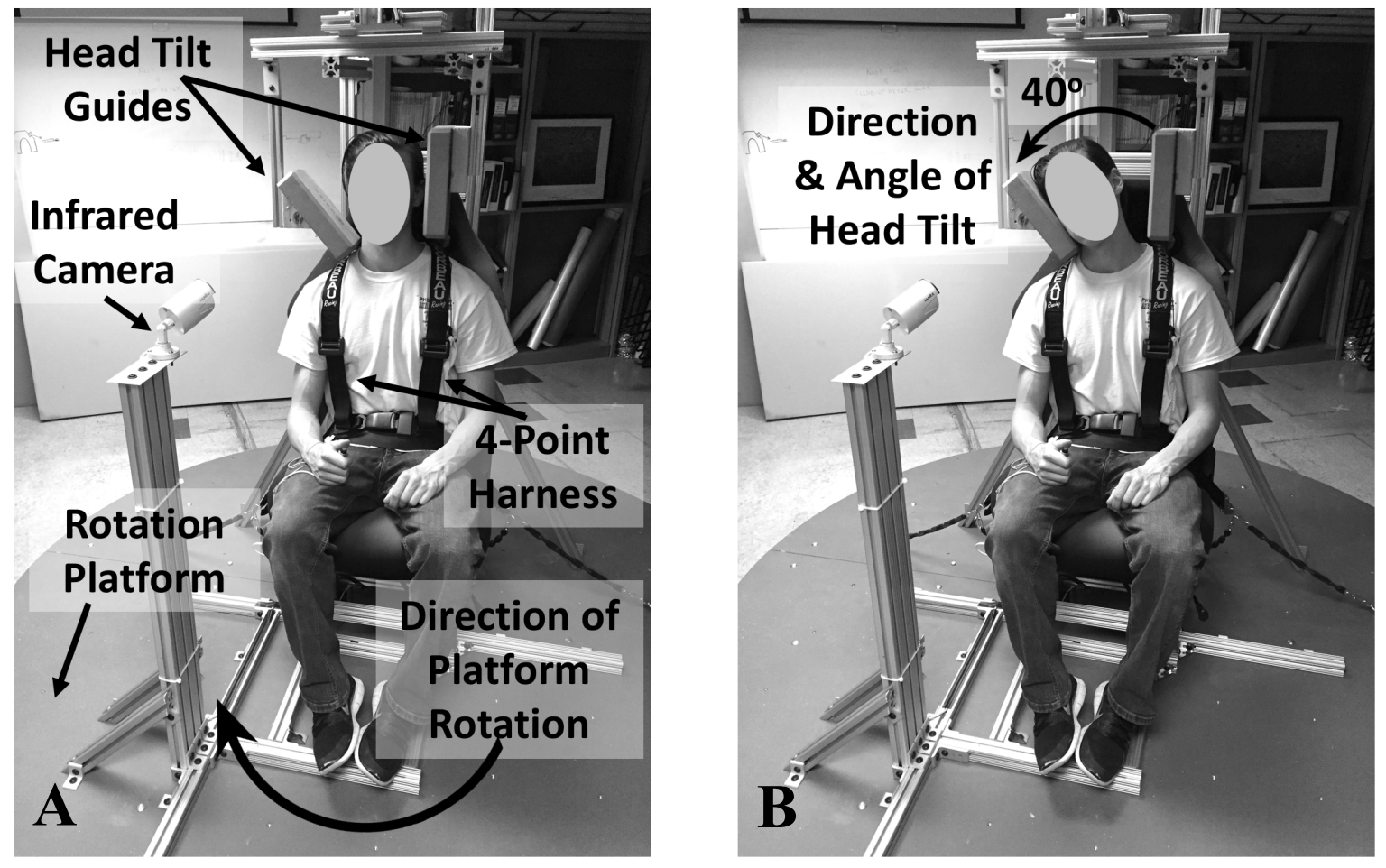
Fig. 2

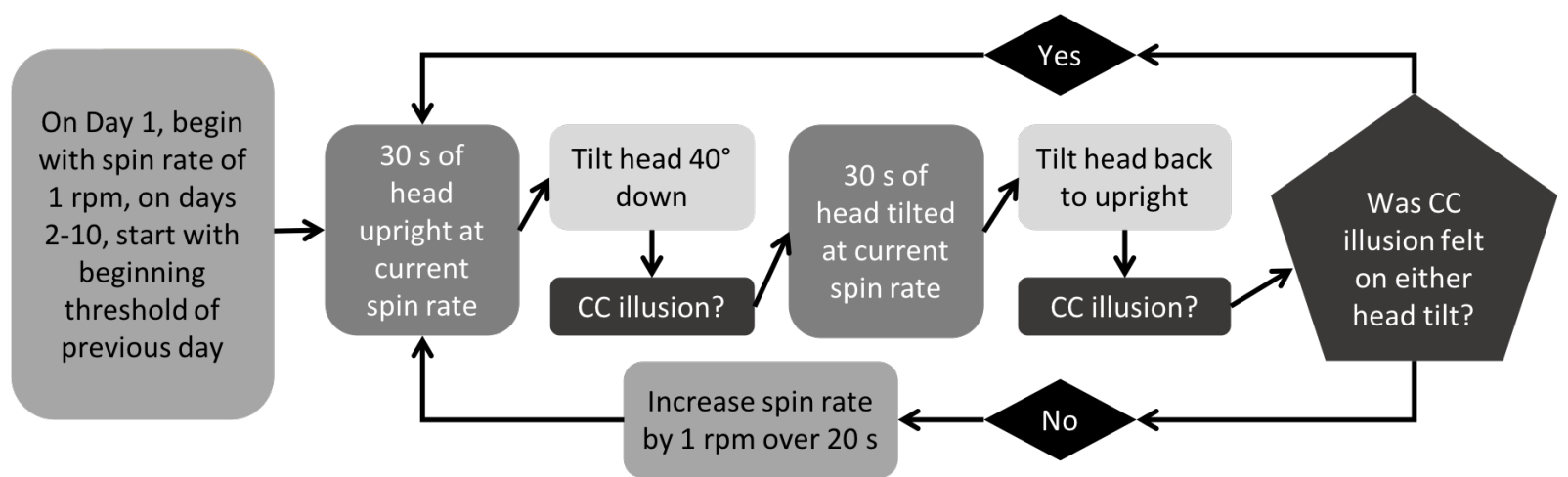


Fig. 3

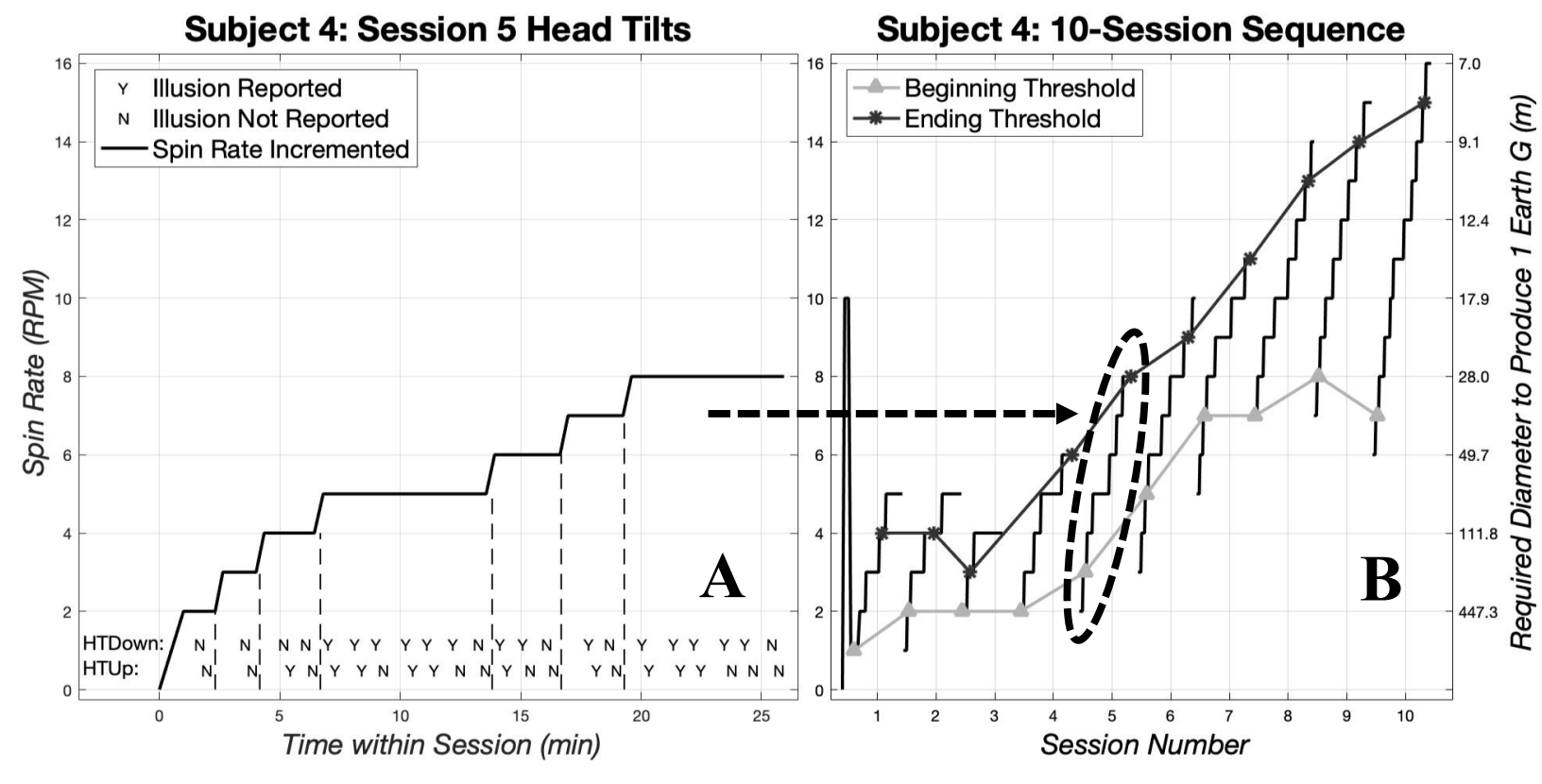


Fig. 4
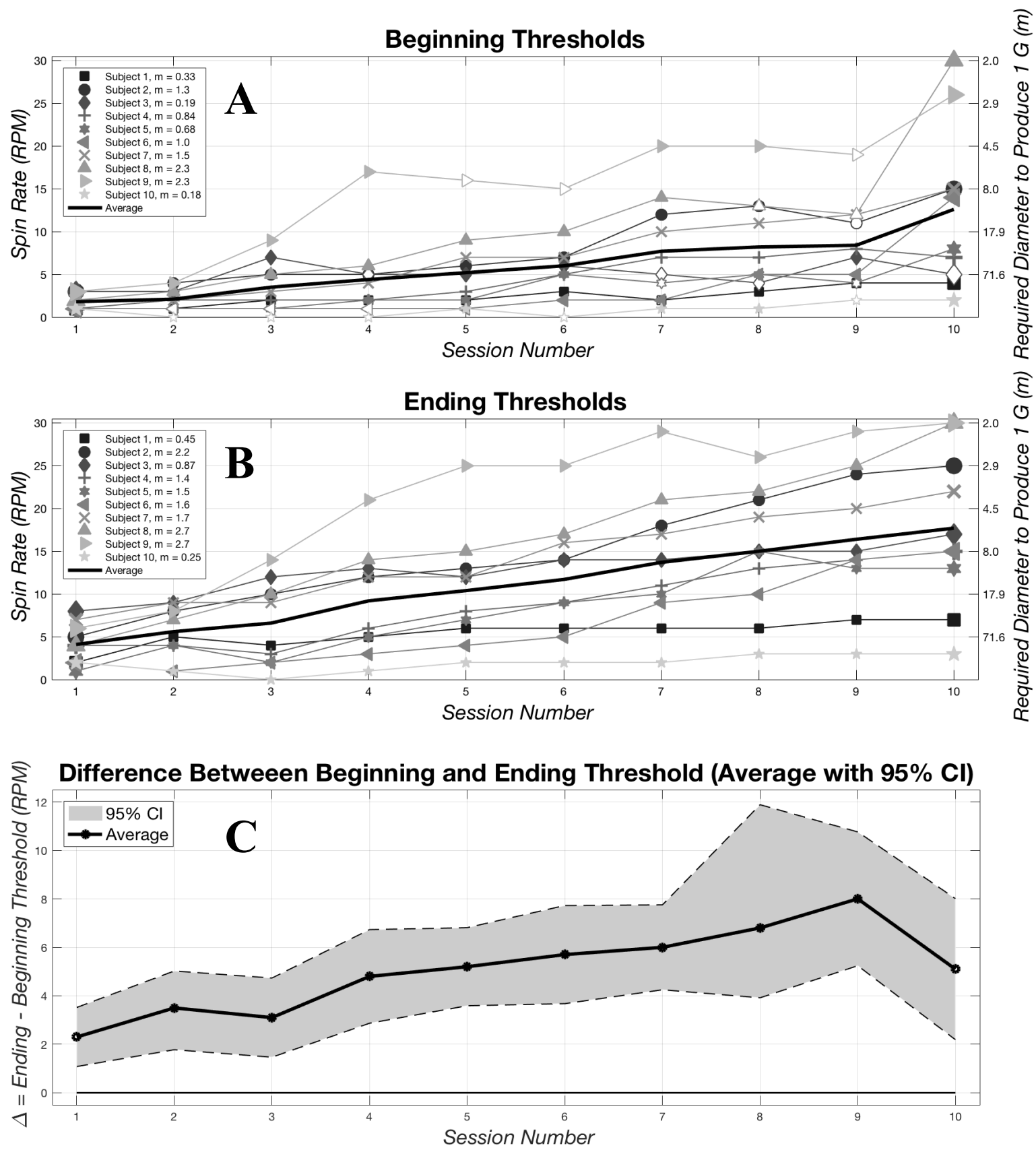
Fig. 5

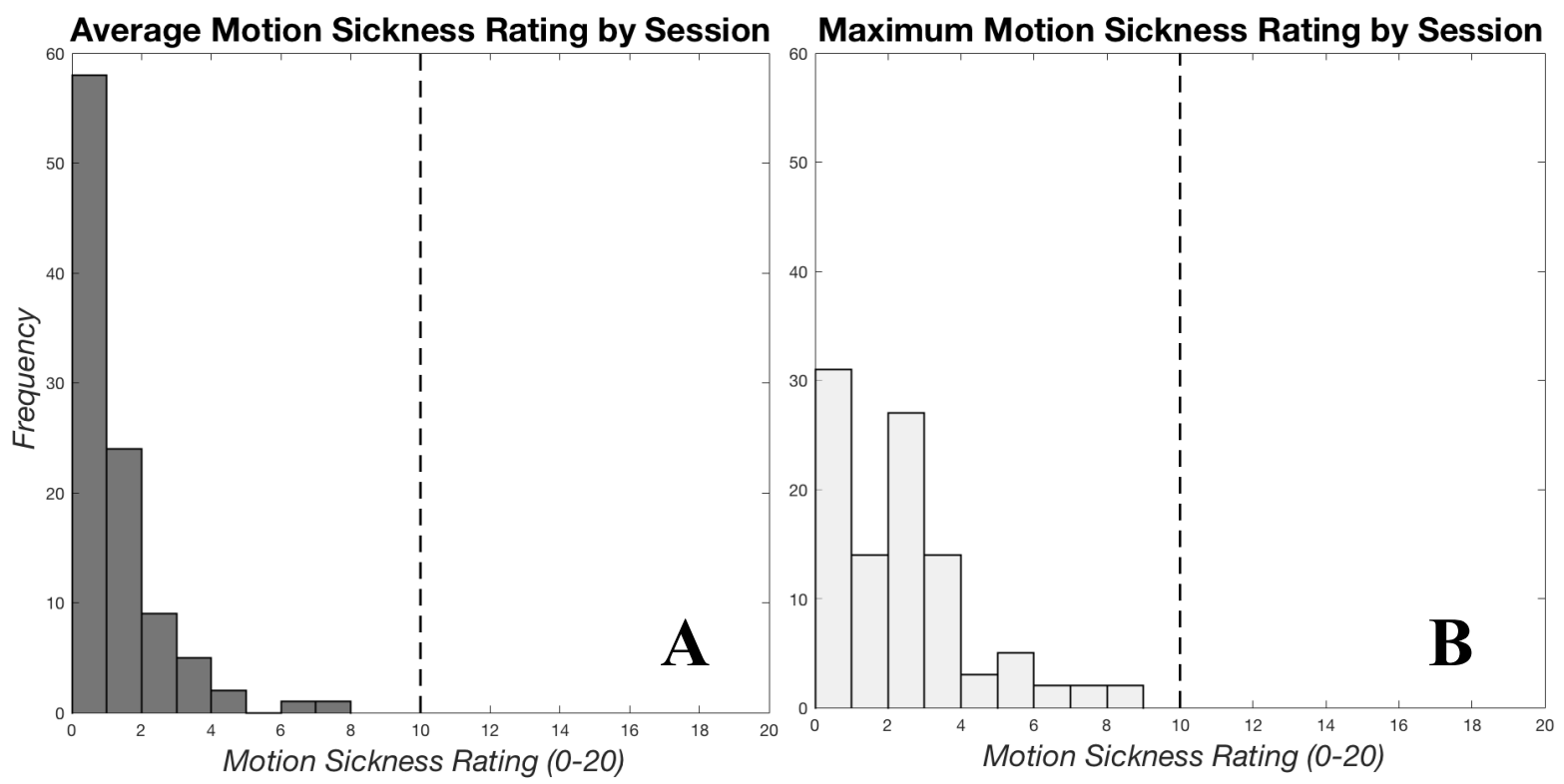

